\title{
Acute Myocardial Infarction as an Initial Symptom of Streptococcus suis Infection: A Case Report
}

\author{
Yi Zhang (D) \\ Xinmei Liu $\mathbb{D}^{2}$ \\ Hongsheng Liu ${ }^{2}$ \\ Chunying Zhang ${ }^{2}$ \\ 'Clinical Medical College of Jining Medical \\ University, Jining, Shandong, 2720I3, \\ People's Republic of China; ${ }^{2}$ Department \\ of Cardiac Intensive Medicine, Affiliated \\ Hospital of Jining Medical University, \\ Jining, Shandong, 272029, People's \\ Republic of China
}

Correspondence: Xinmei Liu Department of Cardiac Intensive Medicine, Affiliated Hospital of Jining Medical University, Jining, Shandong, 272029, People's Republic of China Tel +86 17753825490

Email Ixm19821982@I63.com

\begin{abstract}
Streptococcus suis (S. suis) infection, a zoonotic infection with a global distribution, is clinically manifested by meningitis, followed by sepsis, infective endocarditis and arthritis. S. suis infection is not uncommon, but acute myocardial infarction (AMI), as an initial symptom, has not yet been reported. We report a case of $S$. suis infection with AMI as an initial symptom. The patient, a previously healthy butcher with no known risk factors for AMI, was admitted to hospital with a sudden onset of AMI. Then, thrombolytic therapy, anticoagulation therapy with nadroparin calcium and antiplatelet therapy with aspirin and ticagrelor were adopted. Two days later, blood cultures in aerobic and anaerobic bottles were positive for $S$. suis and he received antibiotic therapy with piperacillin/tazobactam. Then, his symptoms improved and he was transferred to a special infectious disease hospital for further treatment and was discharged upon complete recovery. To the best of our knowledge, this is the first reported case of AMI as the initial symptom of $S$. suis infection, which illustrates a possible new symptom of this important pathogen. For AMI patients with unexplained infections, and who are in close frequent contact with pigs and/or pork products, clinicians should be alert to the possibility of $S$. suis infections.
\end{abstract}

Keywords: Streptococcus suis, acute myocardial infarction, sepsis, zoonosis

\section{Introduction}

Streptococcus suis (S. suis) is a zoonotic pathogen that is transmitted to humans through close contact with infected pigs and/or pork products, which was first reported by Arends and Zanen in Denmark in 1988. ${ }^{1}$ The exact route and mechanism of $S$. suis infection in humans has not been established. Possible transmission routes include inoculation on skin wounds, bioaerosol inhalation and contaminated pork ingestion. $^{2-4}$

S. suis is a facultatively anaerobic Gram-positive cocci that causes meningitis, sepsis, infective endocarditis and arthritis. There exists significant variation in the symptom of $S$. suis infection. Common signs and symptoms include high fever, chills, headache, vomiting, vertigo and rashes. But additionally, hearing loss, walking ataxia, rhabdo myolysis and coma can be observed in patients with meningitis. In patients with toxic septic shock, we can observe hypotension, tachycardia and disseminated intravascular coagulation (DIC). ${ }^{4}$

Previous studies have shown that gram-negative community-acquired bacteremia (CAB) and gram-positive CAB were correlated with risk of AMI. Among them, Streptococcus pneumoniae and Staphylococcus aureus are the representative grampositive cocci, as was Escherichia coli for the gram-negative cocci. ${ }^{5}$ Globally, over 1600 cases of $S$. suis infections have been reported. However, no acute myocardial 
infarction (AMI) associated with S. suis infection has been reported. We report a case of $S$. suis infection-induced AMI, and illustrate a possible new symptom of this important pathogen.

The patient provided informed consent to publish their case details and any accompanying images.

\section{Case Presentation}

A previously healthy 49-year-old male who had been a butcher for 10 years, often injured and wounded his hands at work. He was admitted at a local hospital with a 10-hour acute chest pain that was accompanied by sweating, bilateral lower extremity muscle soreness and limited movements. Upon examination, electrocardiogram (ECG) analysis revealed AMI, as well as hypotension with a blood pressure of 75/36 mmHg. Then, he was administered with thrombolysis therapy. Due to unsatisfactory therapeutic effect, he was referred to our hospital.

Physical examination upon admission: body temperature $37.4{ }^{\circ} \mathrm{C}$; a heart rate of 108 beats $/ \mathrm{min}$, a respiratory rate of 32 breaths/min while his blood pressure was $96 / 67 \mathrm{mmHg}$. Red rash that was fadable by pressing was seen on the shin over the neck and on the upper chest. Heart murmurs were not detected on auscultation and muscle strength testing revealed extremity muscles strength grade 3 . The left little finger of the patient exhibited a cutting wound that was attributed to slaughtering pigs 5 days ago. ECG analysis revealed mild ST-segment elevation and abnormal Q waves in III and aVF; sinus tachycardia and complete right bundle branch block (Figure 1). Transthoracic echocardiography (TTE) analyses revealed suppressed motion amplitude in the basal segments of inferior wall and apical segments without any obvious sign of valve vegetation. Pulmonary computed tomographic angiography (CTA) showed bilateral pleural effusion and inflammation (Figure 2). Laboratory analysis showed an increased white blood cell (WBC) count $\left(44.84 \times 10^{\wedge} 9\right.$ cells/L) with $95.8 \%$ neutrophils, procalcitonin (PCT) at $32 \mathrm{ng} / \mathrm{mL}$, C-reactive protein (CRP) at $170 \mathrm{mg} / \mathrm{L}$, serum creatinine levels of 176.7 umol/L, lactic acid at $3.4 \mathrm{mmol} / \mathrm{L}$, cardiac troponin I (cTnI) level of $24.05 \mathrm{ng} / \mathrm{mL}$, creatine kinase MB (CK-MB) level of $40.3 \mathrm{ng} / \mathrm{mL}$ and myoglobin (MYO) at $118.9 \mathrm{ng} / \mathrm{mL}$, while peripheral blood morphology examination revealed that the abundance of middle and late granulocytes were $3 \%$. On this study, the primary diagnosis was AMI and sepsis. Changes in hemodynamic parameters, temperature, inflammatory and myocardial injury markers during hospitalization are shown in Figures 3-5, respectively.

The patient was quickly admitted to the department of cardiac intensive medicine. Aspirin and ticagrelor were administered in combination with antiplatelet aggregation, nadroparin calcium anticoagulant, rehydration, and papaverine hydrochloride, which improved coronary blood flow. In consideration of unidentified infections, he was

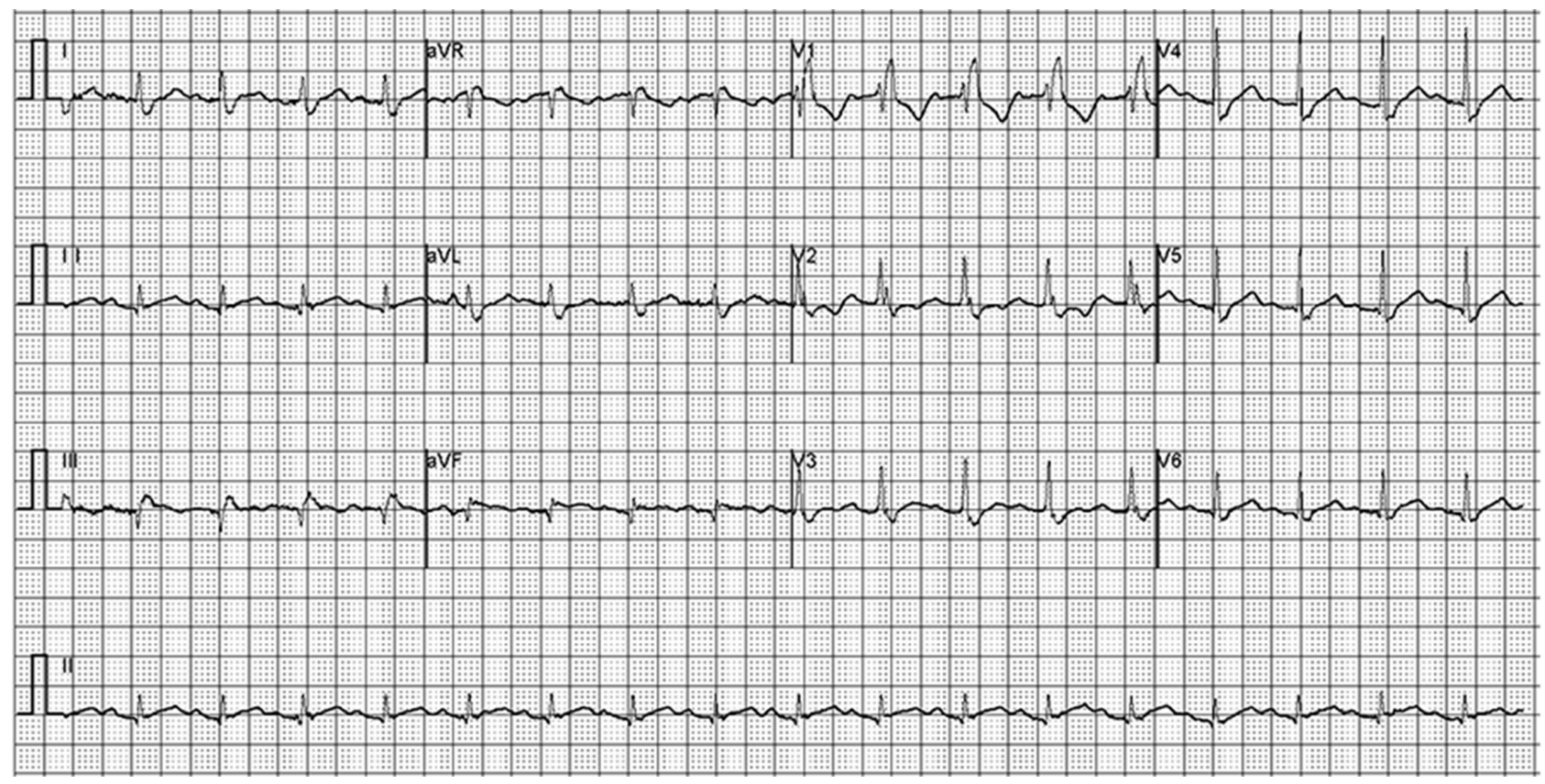

Figure I Electrocardiograph analysis showed mild ST-segment elevation and abnormal Q waves in III and aVF; sinus tachycardia and complete right bundle branch block. 


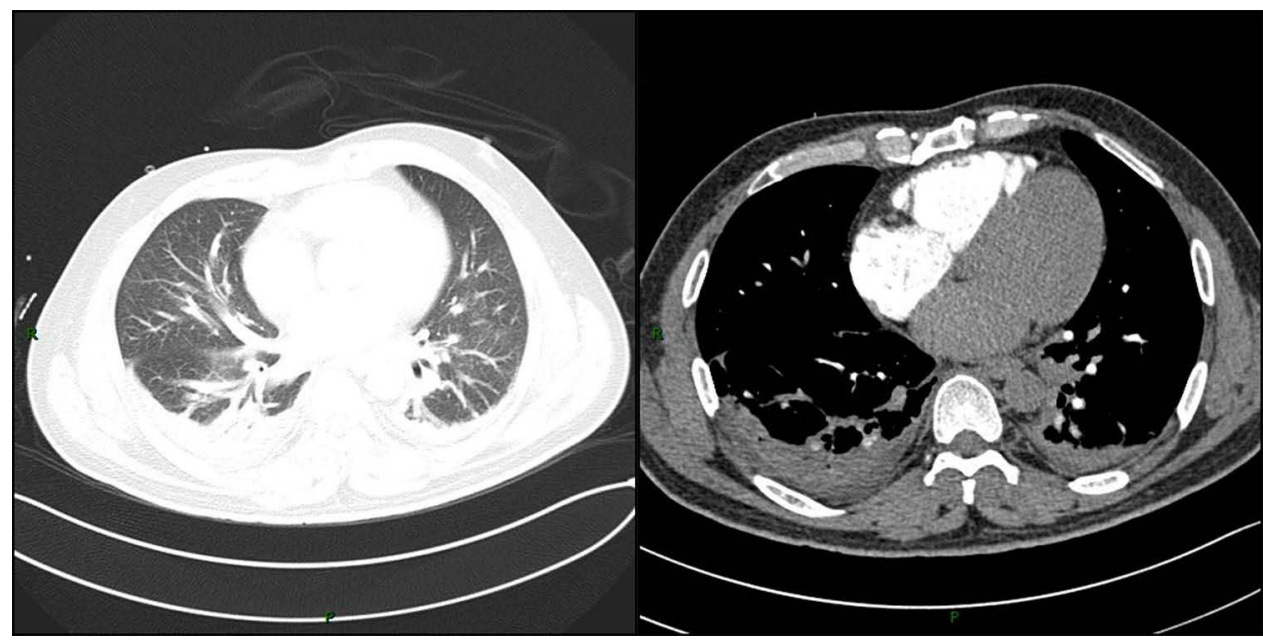

Figure 2 Pulmonary computed tomographic angiography (CTA) showed bilateral pleural effusion and inflammation (pulmonary window and mediastinal window).

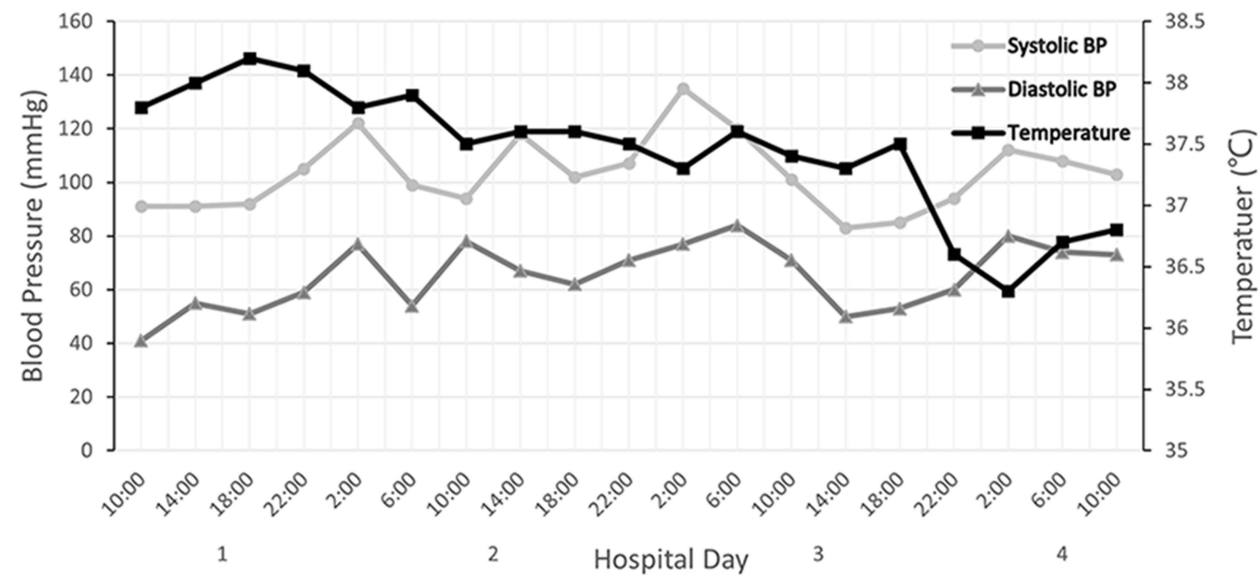

Figure 3 Changes in temperature and blood pressures during hospitalization.

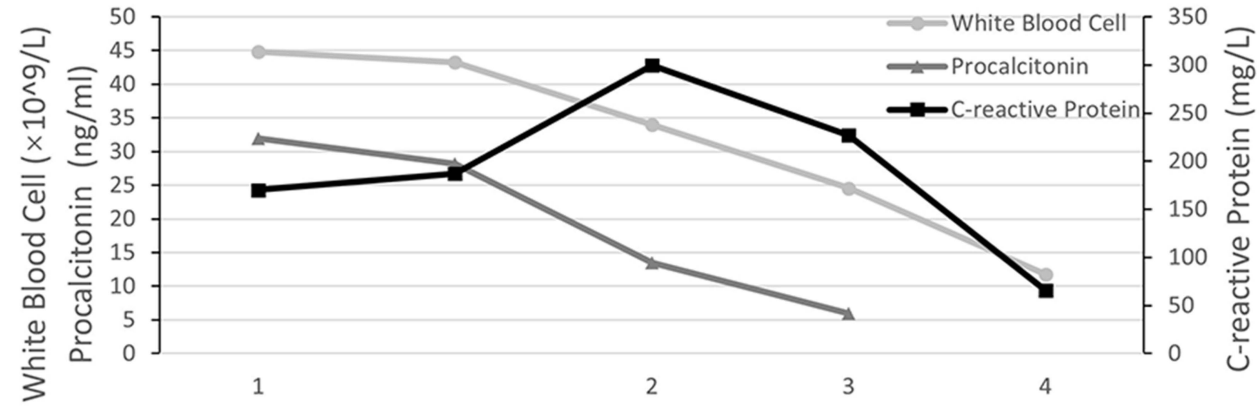

Hospital Day

Figure 4 Changes in inflammatory markers during hospitalization. 


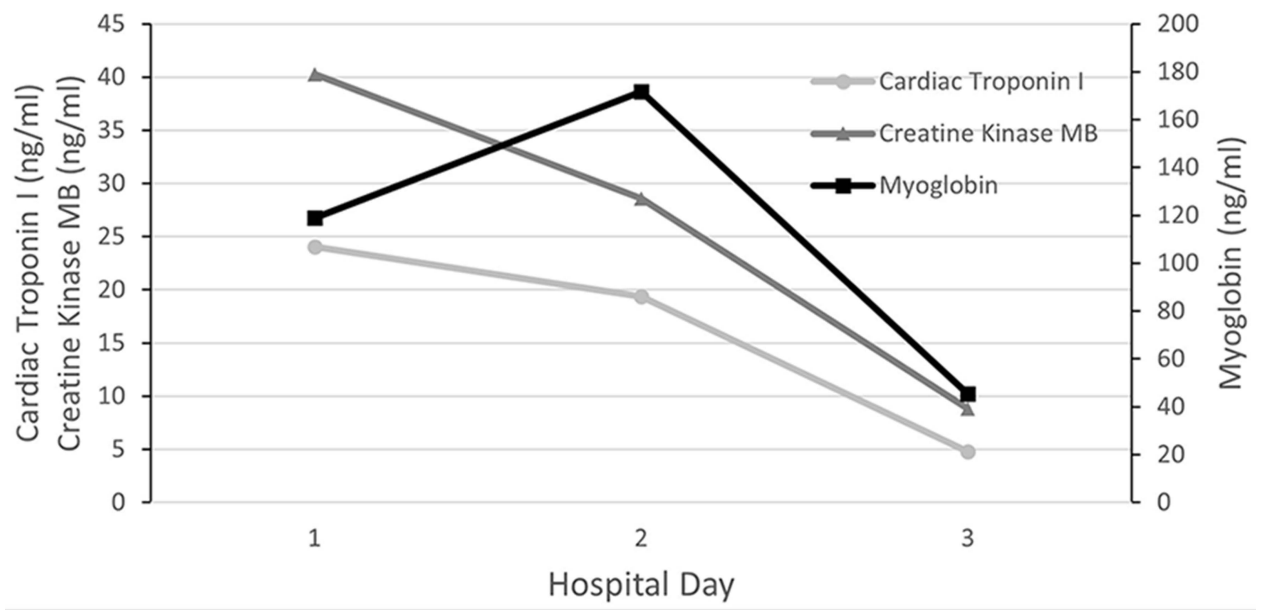

Figure 5 Changes in myocardial injury markers during hospitalization.

instantly empirically intravenously administered with piperacillin/tazobactam $(4.5 \mathrm{~g}$, every $8 \mathrm{~h})$. A day later, his blood pressure had returned to $104 / 64 \mathrm{mmHg}$, but his body temperature fluctuated between 37 and $38{ }^{\circ} \mathrm{C}$. Fortyeight hours later, blood cultures in aerobic and anaerobic bottles were positive for S. suis. Unfortunately, S. suis was not identified at the serotype level due to limited assay conditions. Antimicrobial susceptibility testing revealed sensitivity to ceftriaxone, ampicillin, penicillin, linezolid and levofloxacin, as well as resistance to erythromycin and clindamycin. Based on these findings, we continued with the anti-infection regimen. On day 3 after admission, cTnI, CK-MB and MYO levels decreased to $4.760 \mathrm{ng} / \mathrm{mL}, 8.80$ $\mathrm{ng} / \mathrm{mL}$ and $45.40 \mathrm{ng} / \mathrm{mL}$, respectively, his body temperature decreased to $36.8{ }^{\circ} \mathrm{C}$ and his haemodynamics stabilized. After communicating with family members, he was transferred to a special infectious disease hospital for further treatment on day 4 . His WBC count had decreased to $11.79 \times 10^{\wedge} 9$ cells $/ \mathrm{L}$ with $71.6 \%$ neutrophils when he was transferred to the special infectious disease hospital.

\section{Discussion}

This is a case of middle-aged male with no known risk factors for cardiovascular disorders, presenting with AMI as an initial symptom of $S$. suis infection. In our management the initial priority was to sustain the hemodynamics stable. We also noted that the patient with unidentified infections, he was given antibiotics. The diagnosis of S. suis infection was not made until obtained positive blood culture results.

S. suis is a zoonotic pathogen that is transmitted from infected pigs to humans by close contact. Based on their capsular polysaccharides, S. suis can be classified into 35 serotypes, of which serotype 2 is the most common serotype in human beings worldwide, followed by serotype 14 . $^{4,6}$ S. suis serotype is important in epidemiological studies, to understand the genetic diversity and evolution of capsular polysaccharides in S. suis. ${ }^{7}$ Initially, microbial identification of $S$. suis was done by using biochemical tests. ${ }^{8}$ But because biochemical test results for different types of $S$. suis is significantly varied, other test methods are necessary to type accurately. ${ }^{9}$ With the development of molecular biology technology, polymerase chain reaction (PCR) technology is playing an increasingly essential role in S. suis detection. Neither general PCR nor multiplex PCR (m-PCR) could differentiate certain serotypes. ${ }^{9}$ Matrix-assisted laser desorption/ionization time-of-flight mass spectrometry (MALDI-TOF-MS) has proven to be a reliable identification tool for microbial identification, including $S$. suis identification. ${ }^{8}$ This coupled with PCR technology could improve awareness of the epidemiology and virology of $S$. suis. Unfortunately, we were able to identify S. suis by conventional bacterial culture method only due to the lack of corresponding experience and laboratory equipment. If serotype had been identified, I believe that it would have been more epidemiological implications.

Meningitis is the most common clinical manifestation of $S$. suis infection, followed by sepsis, infective endocarditis and arthritis. ${ }^{10}$ Although S. suis can cause infective endocarditis, no AMI associated with infection has been found so far for $S$. suis.

Acute infection and AMI are both leading causes of short-term mortality worldwide. ${ }^{11}$ Previous studies have 
demonstrated that acute infection may be an acute trigger for cardiovascular events. It commonly involved in AMI include preceding urinary tract and respiratory infections as well as sepsis. ${ }^{12}$ Potential mechanisms of acute infection-induced AMI include demand ischemia, decreasing myocardial contractility and coagulation disturbance. ${ }^{5}$

The majority cases with post-infectious AMI are type 2 MI, which was characterized by insufficient oxygen supply. ${ }^{13}$ Zhang et al ${ }^{14}$ found that there is an important interplay between the $S$. suis suilysin and human platelet, which plays a major role in the process of human platelet activation and aggregation. The process is completed triggered by pore dependent $\mathrm{Ca}^{2+}$ influx, which is fundamental to thrombosis. In our case, the patient had been a butcher by profession for 10 years, and his left little finger had developed a wound 5 days prior to hospital admission. We believe that his severe $S$. suis infection was due to this wound. His chest pain symptoms appeared after finger injury, and he had no known predisposing risk factors for AMI. Combined with the results of previously relevant research and his clinical condition, AMI was therefore considered to have been caused by S. suis infections. But in doing so, we do not have any direct evidence proved that AMI was induced by $S$. suis infection. Of course, whether AMI as a new symptom of this important pathogen needs to be further studied.

$S$. suis is sensitive to many antibiotics. For patients with confirmative $S$. suis infections, antibiotic treatment should be immediately administered. Currently, penicillin $\mathrm{G}$ is recommended as first choice therapeutic option for S. suis infection. ${ }^{4}$ Combinations of penicillin $\mathrm{G}$ with one or more other antibiotics, including ampicillin and ceftriaxone are considered for severely infected patients. ${ }^{415}$ These results were consistent with those of our antimicrobial susceptibility tests. Restoring coronary blood flow, through percutaneous coronary intervention (PCI), is the gold standard for management of conventional acute myocardial infarction (AMI). Based on date from the American National Inpatient Sample database, revascularization was associated with half-reduced in-hospital mortality. ${ }^{16}$ Different points of view also existed in some studies. Putot et al concluded that percutaneous coronary intervention (PCI) might not improve short- and long-term prognosis in patients with significant coronary stenosis. ${ }^{17} \mathrm{But}$ in any case, revascularization may be beneficial for patients who are in medical emergencies. Meanwhile, anti-infection treatment must be used as early as possible. Therefore, we gave anticoagulant treatment along with anti-infection therapy, which led to favorable outcomes.
Despite combinations of antibiotics and other associated treatments, there are some sequelae. Hearing loss, which occurs in $53 \%$ of $S$. suis infected individuals is the most clinical sequelae. It is followed by vestibular dysfunction, ataxia and cognitive impairment. ${ }^{18}$ However, compared to patients without STSS, STSS patients are associated with higher mortality rates, despite adequate treatments. ${ }^{4,19}$ Fortunately, the patient was treated for infection-induced AMI without delay, and he was without sequelae after 3 months of follow-up.

\section{Conclusions}

In summary, S. suis is a significant zoonotic pathogen that is associated with severe life-threatening complications. We describe the first case of AMI as the initial symptom of $S$. suis infection. Systematic education on $S$. suis is the most cost-effective and easiest method for improving the recognition and awareness of this disorder in both patients and physicians. For people in close frequent contact with pigs and/or pork products, the presence of AMI combined with unexplained infections should be taken as possibility of $S$. suis infection. In concurrent AMI treatment, a timely and appropriate empirical antibiotic therapeutic regimen is pivotal to avoid continuous damage.

\section{Abbreviations}

S. suis, Streptococcus suis; AMI, Acute myocardial infarction; ECG, Electrocardiogram; TTE, Transthoracic echocardiography; CT, Computed tomography; WBC, White blood cell; PCT, Procalcitonin; CRP, C-reactive protein; cTnI, Cardiac troponin I; CK-MB, Creatine kinase MB; MYO, Myoglobin; STSS, Streptococcal toxic shock syndrome.

\section{Ethics Approval and Consent to Participate}

Ethics approval for this case report has been provided by the ethics committee of Affiliated Hospital of Jining Medical University.

\section{Acknowledgments}

We appreciate the patient and medical staff members' cooperation in this case report.

\section{Author Contributions}

All authors made substantial contributions to conception and design, acquisition of data, or analysis and interpretation of data; took part in drafting the article or revising it 
critically for important intellectual content; agreed on the journal to which the article will be submitted; gave final approval of the version to be published; and agreed to be accountable for all aspects of the work.

\section{Funding}

This work was supported by Medicine and Health Technology Development Plan Project of Shandong Province (2016WSB34060 to XML), Teacher-Supporting Science Foundation of Jining Medical University (JYFC2018FKJ170 to XML), Key Research and Development Program of Jining Science and Technology Bureau (2020JKNS007 to XML) and Natural Science Foundation of Shandong Province (ZR202102280514 to HSL).

\section{Disclosure}

The authors declare that they have no competing interests.

\section{References}

1. Arends JP, Zanen HC. Meningitis caused by Streptococcus suis in humans. Rev Infect Dis. 1988;10(1):131-137. PMID:3353625. doi:10.1093/clinids/10.1.131

2. Gauthier-Levesque L, Bonifait L, Turgeon N, et al. Impact of serotype and sequence type on the preferential aerosolization of Streptococcus suis. BMC Res Notes. 2016;9:273. PMID:27180230.

3. Feng Y, Zhang H, Wu Z, et al. Streptococcus suis infection: an emerging/reemerging challenge of bacterial infectious diseases. Virulence. 2014;5:477-497. PMID:24667807.

4. Lun ZR, Wang QP, Chen XG, Li AX, Zhu XQ. Streptococcus suis: an emerging zoonotic pathogen. Lancet Infect Dis. 2007;7:201-209. PMID: 17317601.

5. Dalager-Pedersen M, Søgaard M, Schønheyder HC, Nielsen H, Thomsen RW. Risk for myocardial infarction and stroke after community-acquired bacteremia: a 20-year population-based cohort study. Circulation. 2014;129:1387-1396. PMID:24523433.

6. Goyette-Desjardins G, Auger JP, Xu J, Segura M, Gottschalk M. Streptococcus suis, an important pig pathogen and emerging zoonotic agent-an update on the worldwide distribution based on serotyping and sequence typing. Emerg Microbes Infect. 2014;3:e45. PMID:26038745
7. Qiu X, Bai X, Lan R, Zheng H, Xu J. Novel capsular polysaccharide loci and new diagnostic tools for high-throughput capsular gene typing in Streptococcus suis. Appl Environ Microbiol. 2016;82:7102-7112. PMID:27694240.

8. Chaiden C, Jaresitthikunchai J, Kerdsin A, Meekhanon N, Roytrakul S, Nuanualsuwan S. Streptococcus suis serotyping by matrix-assisted laser desorption/ionization time-of-flight mass spectrometry. PLoS One. 2021;16:e249682. PMID:33945547.

9. Xia X, Wang X, Wei X, Jiang J, Hu J. Methods for the detection and characterization of Streptococcus suis: from conventional bacterial culture methods to immunosensors. Antonie Van Leeuwenhoek. 2018;111:2233-2247. PMID:29934695.

10. Huong VT, Ha N, Huy NT, et al. Epidemiology, clinical manifestations, and outcomes of Streptococcus suis infection in humans. Emerg Infect Dis. 2014;20:1105-1114. PMID:24959701.

11. Putot A, Chague F, Manckoundia P, Cottin Y, Zeller M. Postinfectious myocardial infarction: new insights for improved screening. J Clin Med. 2019;8:6.

12. Sebastian S, Stein LK, Dhamoon MS. Infection as a cardiovascular trigger: associations between different organ system infections and cardiovascular events. Am J Med. 2020;133:1437-1443. PMID:32502486.

13. Smilowitz NR, Weiss MC, Mauricio R, et al. Provoking conditions, management and outcomes of type 2 myocardial infarction and myocardial necrosis. Int J Cardiol. 2016;218:196-201.

14. Zhang S, Wang J, Chen S, et al. Effects of suilysin on Streptococcus suis-induced platelet aggregation. Front Cell Infect Microbiol. 2016;6:128

15. Hlebowicz M, Jakubowski P, Smiatacz T. Streptococcus suis meningitis: epidemiology, clinical presentation and treatment. Vector Borne Zoonotic Dis. 2019;19:557-562. PMID:30855223.

16. Liu ES, Chiang CH, Hung WT, et al. Comparison of long-term mortality in patients with acute myocardial infarction associated with or without sepsis. Int $J$ Infect Dis. 2019;79:169-178.

17. Putot A, Chagué F, Manckoundia P, et al. Post-infectious myocardial infarction: does percutaneous coronary intervention improve outcomes? A propensity score-matched analysis. J Clin Med. 2020;9:6.

18. van Samkar A, Brouwer MC, Schultsz C, van der Ende A, van de Beek D. Streptococcus suis Meningitis: a systematic review and meta-analysis. PLoS Negl Trop Dis. 2015;9:e0004191. PMID:26505485.

19. Rayanakorn A, Goh BH, Lee LH, Khan TM, Saokaew S. Risk factors for Streptococcus suis infection: a systematic review and meta-analysis. Sci Rep. 2018;8:13358. PMID:30190575.
International Medical Case Reports Journal

\section{Publish your work in this journal}

The International Medical Case Reports Journal is an international, peer-reviewed open-access journal publishing original case reports from all medical specialties. Previously unpublished medical posters are also accepted relating to any area of clinical or preclinical science. Submissions should not normally exceed 2,000 words or 4 published pages including figures, diagrams and references. The manuscript management system is completely online and includes a very quick and fair peer-review system, which is all easy to use. Visit http://www.dovepress.com/testimonials.php to read real quotes from published authors. 